\title{
Assessment of Robot-Assisted Surgery in a Children's Hospital by applying the "DoHTA" Method
}

\begin{abstract}
Robotic surgery (RS) has been proposed as a minimally invasive surgical technique with advantages for both surgeons and patients, but it is concurrently associated with high costs. This paper shows the results of the application of Decision-oriented Health Technology Assessment (doHTA) method to assess the potential implementation of robotic surgical system compared to laparoscopic surgery (LS) and open surgery (OS). DoHTA is a new implementation of EUnetHTA Core Model ${ }^{\circledR}$, which integrates the Multi-Criteria Decision Analysis (MCDA) by using the Analytic Hierarchy Process (AHP). The analysis took into account recommendations for robotic surgery gathered from scientific literature, experts' judgments and hospital management data.

The assessment revealed the feasibility, safety, technical characteristics, efficacy, and negative economic impact (mainly due to the high purchase costs) of RS. Based on doHTA results, the estimated performances of RS have turned out to be comparable with LS with respect to safety and clinical effectiveness, but about seven times and three times worse in terms of economic and organizational performances, respectively. Accordingly, the RS ranked $3^{\text {rd }}$ in the final ranking. The sensitivity analysis shows that the final ranking is sufficiently robust to affirm that the adoption of RS is at this time unfeasible of adoption within our hospital.
\end{abstract}

Keywords: Health Technology Assessment, doHTA, Robotic-Assisted Surgery

\section{Introduction}

Over the last few years, the number of robotic surgical procedures has had a significant increase. The decision to adopt the robotic system (basing that decision on the existing evidence) is still difficult to realize as it can be used for different indications for which evidence is limited, and the evaluation of its costs generally depends on its use.

\section{Literature review}

Many studies and HTA reports analyzed the potential impact of RS on different clinical outcomes; it appears to favor RS compared to LS and OS. However, RS does not seems to provide a strong basis for recommendations capable of directing the assessment process of RS and the decision makers' final choice. 


\section{Hypotheses/Objectives}

The goal of this study is to develop a comprehensive assessment of RS compared to LS and OS in order to support the final decision whether to undertake the robotic system in the hospital.

\section{Research Design/Methodology}

The assessment of RS in a range of pediatric surgical procedures was performed by using DoHTA method, which integrates the EUnetHTA CoreModel $\mathbb{C}$ and Analytic Hierarchy Process (AHP). A decision tree covering all the relevant assessment aspects (e.g. safety, efficacy, costs) of robotic surgery has been derived and weighted by means of pairwise comparisons. The combination of KPIs and AHP gave the opportunity to model experts' judgments, supplementing the limited literature evidence. Applying sensitivity analysis, we have investigated the effect of uncertainty of judgments on the stability of rank order of the alternatives. The sensitivity analysis has ensured the consistency of final decision, empowering decision makers to choose more knowingly among different alternative technologies.

\section{Results}

DoHTA results show that the estimated performance of RS have turned out to be comparable to LS pertaining to safety and clinical effectiveness domains, but about six times and three times worse in terms of economic and organizational performances, respectively, thus posing $\mathrm{RS}$ at $3^{\text {rd }}$ place in the final ranking. The sensitivity analysis results show that safety and clinical effectiveness domains are the most critical criteria, whereas the most critical measure of performance pertains the technical features of the considered surgical techniques.

\section{Limitations}

Potential bias resulting from the doHTA application to RS pertain the limited sample of professionals who have worked to the assessment. To overcome such issue, it is essential to involve all those professionals representing hospital sectors potentially affected by the implementation of RS.

\section{Conclusions}

According to doHTA results, RS seems to offer some advantages for both patients and surgeons (e.g. better precision, articulations of instruments and three-dimensional visualization). Nonetheless, it is currently difficult to make up for the initial investment with RS: it implies increased hospital costs (e.g. capital acquisition cost, service costs, and costs of operations, which often exceeded the tariffs for reimbursement) compared to its alternatives. Sensitivity analysis also confirmed that final alternatives' ranking is 
ISAHP Article: A Style Guide for Paper Proposals To Be Submitted to the International Symposium on the Analytic Hierarchy Process 2016, London, U.K.

consistent and robust; such considerations have led to the decision that the adoption of $\mathrm{RS}$ is currently unfeasible in our hospital.

\section{Key References}

- $\quad$ Ritrovato M, Faggiano FC, Tedesco G, Derrico P. Decision-Oriented Health Technology Assessment: One Step Forward in Supporting the Decision-Making Process in Hospitals. Value Health. 2015 Jun;184:505-11

- $\quad$ Saaty TL. The Analytic Hierarchy Process. New York: McGraw Hill, 1980.

- Tedesco G, Faggiano FC, Leo E, Derrico P, Ritrovato M. A comparative cost analysis of robotic-assisted surgery versus laparoscopic surgery and open surgery: the necessity of investing knowledgeably. Surg Endosc. 2016 Mar 16. [Epub ahead of print] 